\title{
BMJ Open Impact of intrapatient variability (IPV) in tacrolimus trough levels on long-term renal transplant function: multicentre collaborative retrospective cohort study protocol
}

\author{
Petra M Goldsmith, ${ }^{1,2}$ Matthew J Bottomley, ${ }^{3}$ Okidi Okechukwu, ${ }^{4}$ Victoria C Ross, ${ }^{5}$ \\ Ryan Ghita, ${ }^{5}$ David Wandless, ${ }^{6}$ Stuart J Falconer, ${ }^{7}$ Stavros Papachristos, ${ }^{4}$ \\ Philip Nash, ${ }^{8}$ Vitaliy Androshchuk, ${ }^{3}$ Marc Clancy, ${ }^{7,9}$ Transplant Audit Collaborative
}

To cite: Goldsmith PM, Bottomley MJ, Okechukwu 0, et al. Impact of intrapatient variability (IPV) in tacrolimus trough levels on long-term renal transplant function: multicentre collaborative retrospective cohort study protocol. BMJ Open 2017;7:e016144. doi:10.1136/ bmjopen-2017-016144

- Prepublication history for this paper is available online. To view these files please visit the journal online (http://dx.doi. org/10.1136/bmjopen-2017016144).

Received 27 January 2017

Revised 5 June 2017

Accepted 9 June 2017

\section{ABSTRACT}

Introduction High intrapatient variability (IPV) in tacrolimus trough levels has been shown to be associated with higher rates of renal transplant failure. There is no consensus on what level of IPV constitutes a risk of graft loss. The establishment of such a threshold could help to guide clinicians in identifying at-risk patients to receive targeted interventions to improve IPV and thus outcomes. Methods and analysis A multicentre Transplant Audit Collaborative has been established to conduct a retrospective study examining tacrolimus IPV and renal transplant outcomes. Patients in receipt of a renal transplant at participating centres between 2009 and 2014 and fulfilling the inclusion criteria will be included in the study. The aim is to recruit a minimum of 1600 patients with follow-up spanning at least 2 years in order to determine a threshold IPV above which a renal transplant recipient would be considered at increased risk of graft loss. The study also aims to determine any national or regional trends in IPV and any demographic associations. Ethics and dissemination Consent will not be sought from patients whose data are used in this study as no additional procedures or information will be required from participants beyond that which would normally take place as part of clinical care. The study will be registered locally in each participating centre in line with local research and development protocols. It is anticipated that the results of this audit will be disseminated locally, in participating NHS Trusts, through national and international meetings and publications in peer-reviewed journals.

\section{INTRODUCTION}

The addition of calcineurin inhibitors (CNIs) as maintenance immunosuppressants has improved renal transplant 1-year survival rates since the 1980s. ${ }^{1}$ Tacrolimus emerged as a viable alternative to ciclosporin in the 1990s. ${ }^{1}$ In 2005, a meta-analysis was published on randomised trial data comparing tacrolimus and ciclosporin as primary
Strengths and limitations of this study

- This is a multicentre collaborative study composed of units across the UK which are both transplant centres and referring nephrology units.

- The sample size will be the largest investigating IPV to date.

- The study uses a unified method across all participating centres, which will enable meaningful comparison between centres.

- The study investigates retrospective data and requires a lengthy follow-up period which will lead to some exclusions.

- There will be minor local variations in the laboratory assay that cannot be corrected for in the IPV calculations

- Confounding factors such as geographical bias, repatriation as a cause of lost to follow-up, underrepresentation of poorly compliant patients who do not attend appointments, frequency of sampling and temporary medications are beyond the scope of this study.

immunosuppressants in renal transplant, observing a $44 \%$ reduction in death-censored graft loss with tacrolimus over ciclosporin. ${ }^{2}$ In 2007, the Symphony Study reported favourable graft survival and function, and reduced biopsy-proven rejection with low-dose tacrolimus over low-dose ciclosporin, sirolimus or standard-dose tacrolimus. ${ }^{3}$

CNIs have a narrow therapeutic index: too little exposure places a transplant recipient at increased risk of acute rejection and donor-specific antibody formation. Too much exposure and a transplant recipient is placed at increased risk of malignancy, infection, nephrotoxicity and unacceptable side effects such as tremor. ${ }^{4-7}$ 
Trough levels are used as a proxy for oral bioavailability of CNIs and vary both between patients (interpatient variability) and for an individual over time (intrapatient variability, IPV). Between individuals, age, gender, ethnicity, body mass index, genetic polymorphisms in CYP3A5 and CYP3A4, drug interactions, adherence, liver function and lifestyle choices account for the differences. Similarly, IPV is affected by adherence, gastrointestinal metabolism and motility, diarrhoea, food and drug interactions, synchronicity of dose administration and blood test and variability of the laboratory assay. ${ }^{8-15}$

An emerging body of evidence is being established indicating favourable graft function, survival and fewer rejection episodes up to 1 year post-transplant for patients demonstrating low IPV. ${ }^{16-18}$ Similarly, high IPV has been associated with poorer outcomes and graft survival. ${ }^{19} 20$ Donor age and previous transplants appear to be risk factors for a high IPV. ${ }^{18}$ However, little data exists on the long-term impact of high IPV and studies have not yet been able to draw conclusions about risk thresholds of variability because of limitations in sample size.

\section{Objectives}

- To establish important baseline data about national and regional trends in IPV

- To investigate demographic associations and other characteristics for patients in high and low variability groups

- To establish whether there exists a 'danger' threshold for IPV, above which a patient is deemed at risk of graft loss or dysfunction, so they can then be targeted for intervention prior to organ damage or failure

\section{Outcomes}

Primary outcomes: Recent 12 months' IPV, IPV months 6-12, change in IPV.

Secondary outcomes: Ethnicity, recipient age, change in IPV, previous transplants, DR mismatch, graft function, graft survival, gender.

\section{Confounders}

We acknowledge the potential for confounding factors that are outside the scope of this study to address. These include frequency of tacrolimus level sampling (and the reasons why this might be increased) and conversely under-representation of poorly compliant patients who do not attend appointments. These confounders may be affected by hospital admissions, temporary medication use (such as oral antibiotics) and those patients with a modified tacrolimus target.

\section{METHODS AND ANALYSIS}

A multicentre transplant audit collaborative (TAC) has been established to conduct a retrospective study examining tacrolimus IPV and renal transplant outcomes. It is the first collaborative of its kind, facilitating the development of this largest study examining IPV to date. TAC is composed of junior doctors with an interest in
Table 1 Inclusion/exclusion criteria

Inclusion criteria Exclusion criteria

Age $\geq 18$ years at time Age $\leq 17$ years at time of transplant of transplant

A functioning graft* Failed graft before 2 years at 2 years

\begin{tabular}{ll}
$\begin{array}{l}\text { Renal-only } \\
\text { transplant }\end{array}$ & Non-renal transplant \\
$\begin{array}{l}\text { Short-acting } \\
\text { tacrolimus } \\
\text { preparation }\end{array}$ & Modified release preparation at any \\
$\begin{array}{l}\text { Only during study } \\
\text { period }\end{array}$ & Point during study period \\
\hline $\begin{array}{l}\text { Tacrolimus } \\
\text { as primary } \\
\text { immunosuppressant }\end{array}$ & $\begin{array}{l}\text { Other primary immunosuppressant } \\
\text { (eg, ciclosporin, sirolimus, other non- } \\
\text { steroid) }\end{array}$ \\
$\begin{array}{l}\text { Alive minimum } \\
2 \text { years following } \\
\text { transplant }\end{array}$ & $\begin{array}{l}\text { Pregnancy at any point during study } \\
\text { period }\end{array}$ \\
& $\begin{array}{l}\text { Death prior to 2years following } \\
\text { transplant }\end{array}$ \\
& $\begin{array}{l}\text { Non-standard transplant (ABO } \\
\text { or HLA incompatible; requiring } \\
\text { desensitisation) }\end{array}$ \\
& $\begin{array}{l}\text { Patients with fewer than four } \\
\text { tacrolimus trough levels for T1 and T2 }\end{array}$ \\
\hline
\end{tabular}

T1, 6-12 months post-transplant; T2, most recent 12 months.

nephrology and/or transplantation. It is supported by Consultant physicians and surgeons in these fields to undertake research and audit projects related to transplantation. Any UK NHS Trust involved in the aftercare of renal transplant recipients is eligible to register for this study providing they have a transplant or nephrology doctor willing to enrol in the TAC.

\section{Patient selection}

Patients in receipt of a renal transplant at participating centres between 2009 and 2014 and fulfilling the inclusion criteria will be included in the study (table 1). To be enrolled, patients are required to have follow-up spanning at least 2 years in order to determine a threshold IPV above which a renal transplant recipient would be considered at increased risk of graft loss.

\section{Sample size}

A large sample size is needed to provide meaningful numbers to establish variability risk cut-offs. It is estimated that a minimum of eight UK-based centres will participate in the study. If each centre, on average, supplies data for 200 patients, a minimum dataset of 1600 patients will be achieved.

It is, however, recognised that there will be a significant variation between the numbers of eligible patients available to each centre (tertiary transplant units will naturally have access to larger numbers that referring District General Hospitals). The set number of 200, therefore, is 


\section{Box 1 Minimum dataset}

Data set
Date of transplant
Recipient and donor ages
Gender
Ethnicity
Primary renal diagnosis
Previous transplants
Mismatch grade (A, B, DR)
Type of donor
- eGFR at the end of $\mathrm{T} 1$ and $\mathrm{T} 2$
Creatinine at 12 months and at the end of $\mathrm{T} 2$
All tacrolimus trough levels during $\mathrm{T} 1$ and $\mathrm{T} 2$
Urinary protein creatinine ratio at the of end $\mathrm{T} 1$ and $\mathrm{T} 2$
Graft failure
Delayed graft function
Tacrolimus dosing at month 12 and at the end of $\mathrm{T} 2$
Other immunosuppression at both $\mathrm{T} 1$ and $\mathrm{T} 2$ :
- Steroid $\mathrm{Y} / \mathrm{N} ;$
- MMF $\mathrm{Y} / \mathrm{N} ;$
- Azathioprine Y/N
Induction agent
De novo donor-specific antibody status post-transplant and level
(mean fluorescence intensity)
Biopsy-proven acute rejection
Death

T1, 6-12 months post-transplant; T2, most recent 12 months.

only to be used as a guide and it is recognised that this may not be achievable for smaller, secondary nephrology units. Similarly, we welcome larger numbers from any centre able to do so.

\section{Data collection and information governance}

TAC has established a standard minimum dataset (see box 1) which each site will use as the basis for data collection. A template database will be provided to a representative of the study team from each NHS Trust participating in the study. This database will be password protected and held on secure local Trust servers. All data should be entered onto this database - the use of hard copy proformas associated with this audit is discouraged to avoid breaching data protection policies.

The data collection database will be anonymised and will contain no patient identifiable data. Each hospital will be issued with patient study identifiers for all patients included in the study. A separate password-protected spreadsheet of the study identifiers with the corresponding hospital numbers should be securely stored locally by each participating centre for local reference only should there be any difficulties or queries regarding data collection.

The anonymised master database will be compiled by a dedicated, named member of the collaborative. It will be shared with all members of TAC using secure NHS email only (either local Trust email or NHS.net) and will be held on secure, password-protected Trust servers only.

Data collection has been under way since March 2017 and is scheduled for completion in September 2017. A period of data analysis will then follow until December 2017 at which time the results will be disseminated as detailed in the Ethics and Dissemination section below.

\section{Data analysis}

Studies have shown that high IPV is associated with poorer renal transplant outcomes ${ }^{17}$ but no research group has yet established or described an IPV level at which the risk of such outcomes is significantly increased. With our large sample size, we will be able to stratify our group into quartiles or quintiles based on IPV, enabling us to compare outcomes between the groups.

IPV will be calculated using the mean absolute deviation as described by Shuker et al. ${ }^{18}$ Individual subjects will be stratified into groups based on observed variability during T1. Intergroup comparisons will be made using both univariate and multivariate analyses for the clinically relevant end points including graft loss, graft dysfunction (assessed by eGFR and new onset proteinuria) and biopsy-proven rejection episodes. The univariate predictive value of T1 IPV for these outcomes will be evaluated by receiver operator curve assessment.

Categorical variables will be compared using $\mathrm{X}^{2}$ and Fisher's exact test where appropriate. Continuous variables will be assessed using $\mathrm{T}$ test for parametric and Mann-Whitney U test for non-parametric data.

Multivariate analysis will use Cox regression survival analysis to compare event-free survival, corrected for potential confounders including age, gender and ethnicity. Where there is loss to follow-up after the 2-year period, data will be censored according to last known status at the time of last creatinine or tacrolimus level (whichever is the latter).

\section{Permissions and registration}

Each participating centre will be expected to complete a site registration form. A named member of TAC from each participating centre will be responsible for gaining all necessary local Trust permissions and study registrations as required by local Research and Development and Audit offices.

Consent will not be sought from patients whose data are used in this study as no additional procedures or information will be required from participants beyond that which would normally take place as part of clinical care. The findings of the study are not expected to impact on individual patient care.

\footnotetext{
Author affiliations

${ }^{1}$ Renal Transplant Unit, Royal Liverpool University Hospitals NHS Trust, Liverpool, UK ${ }^{2}$ Institute of Infection and Global Health, University of Liverpool, Liverpool, UK ${ }^{3}$ Department of Nephrology, Oxford University Hospitals NHS Foundation Trust, Oxford, UK

${ }^{4}$ Department of Transplantation, Central Manchester University Hospitals NHS Foundation Trust, Manchester, UK

${ }^{5}$ Department of Transplantation, NHS Greater Glasgow and Clyde, Glasgow, UK ${ }^{6}$ Department of Nephrology, NHS Grampian, Aberdeen, UK

${ }^{7}$ Department of Transplantation, NHS Lothian, Edinburgh, UK

${ }^{8}$ Department of Nephrology, King's College Hospital NHS Foundation Trust, London, UK

${ }^{9}$ School of Medicine, Dentistry and Surgery, University of Glasgow, Glasgow, UK
} 
Contributors PMG wrote the protocol, produced revisions and was involved in the original concept, study design and implementation. MJB made substantial revisions to the protocol and was involved in the original concept, study design and implementation. 00 made substantial revisions to the protocol and was involved in the original concept, study design and implementation. VCR made revisions to the protocol and was involved in the original concept, study design and implementation. $\mathrm{RG}$ made revisions to the protocol and was involved in the original concept, study design and implementation. DW made revisions to the protocol and was involved in the original concept, study design and implementation. SJF made revisions to the protocol and was involved in the original concept, study design and implementation. SP made revisions to the protocol and was involved in the original concept, study design and implementation. PN made revisions to the protocol and was involved in the original concept, study design and implementation. VA made revisions to the protocol and was involved in the original concept, study design and implementation. $\mathrm{MC}$ is senior author and made revisions to the protocol and was involved in the original concept, study design and implementation.

Funding Funding for this publication will be provided by Astellas Pharma UK.

Competing interests The Transplant Audit Collaborative acknowledges financial support from Astellas Pharma UK in the facilitation of meetings, in the provision of training opportunities for its members and in funding publication costs. Astellas Pharma UK has been given no editorial capacity in this paper, neither will they be given access to raw data nor influence any aspect of the study which will be conducted transparently and without bias. Astellas Pharma UK will not be involved in the study design, interpretation of data or in the authorship of disseminations arising from the work being undertaken.

Patient consent Detail has been removed from this case description/these case descriptions to ensure anonymity. The editors and reviewers have seen the detailed information available and are satisfied that the information backs up the case the authors are making.

Ethics approval As this study does not fulfil the NHS Health Research Authority and Medical Research Council's criteria for research, formal ethical approval is not needed. However, all local NHS Trust approvals and registrations will be sought from each participating centre. It is anticipated that the results of this audit will be disseminated locally, in participating NHS Trusts, through national and international meetings and publications in peer-reviewed journals.

Provenance and peer review Not commissioned; externally peer reviewed.

Data sharing statement At the time of writing this protocol, data has not been collected and thus data sharing is not applicable at this time.

Open Access This is an Open Access article distributed in accordance with the Creative Commons Attribution Non Commercial (CC BY-NC 4.0) license, which permits others to distribute, remix, adapt, build upon this work non-commercially, and license their derivative works on different terms, provided the original work is properly cited and the use is non-commercial. See: http://creativecommons.org/ licenses/by-nc/4.0/

(c) Article author(s) (or their employer(s) unless otherwise stated in the text of the article) 2017. All rights reserved. No commercial use is permitted unless otherwise expressly granted.

\section{REFERENCES}

1. Hong JC, Kahan BD. Immunosuppressive agents in organ transplantation: past, present, and future. Semin Nephrol 2000;20:108-25.
2. Webster AC, Woodroffe RC, Taylor RS, et al. Tacrolimus versus ciclosporin as primary immunosuppression for kidney transplant recipients: meta-analysis and meta-regression of randomised trial data. BMJ 2005:331:810.

3. Ekberg H, Tedesco-Silva $\mathrm{H}$, Demirbas $\mathrm{A}$, et al. Reduced exposure to calcineurin inhibitors in renal transplantation. N Engl J Med Overseas Ed 2007;357:2562-75.

4. Hesselink DA, van Gelder T, van Schaik RH, et al. The pharmacogenetics of calcineurin inhibitors: one step closer toward individualized immunosuppression? Pharmacogenomics 2005;6:323-37.

5. http://www.ema.europa.eu/docs/en GB/document library/EPAR Product_Information/human/000712/WC500022234.pdf3EMA: EMA/618604/2008. (10 Dec 2012)

6. Schiff J, Cole E, Cantarovich M, et al. Therapeutic monitoring of calcineurin inhibitors for the nephrologist. Clin J Am Soc Nephrol 2007;2:374-84

7. Venkataramanan R, Swaminathan A, Prasad T, et al. Clinical pharmacokinetics of tacrolimus. Clin Pharmacokinet 1995;29:404-30.

8. Antignac M, Barrou B, Farinotti R, et al. Population pharmacokinetics and bioavailability of tacrolimus in kidney transplant patients. $\mathrm{Br} \mathrm{J}$ Clin Pharmacol 2007;64:750-757.

9. de Jonge $\mathrm{H}$, Naesens $\mathrm{M}$, Kuypers DR, et al. New insights into the pharmacokinetics and pharmacodynamics of the calcineurin inhibitors and mycophenolic acid: possible consequences for therapeutic drug monitoring in solid organ transplantation. Ther Drug Monit 2009;31:416-35.

10. Scholten EM, Cremers SC, Schoemaker RC, et al. AUC-guided dosing of tacrolimus prevents progressive systemic overexposure in renal transplant recipients. Kidney Int 2005;67:2440-7.

11. Dew MA, DiMartini AF, De Vito Dabbs A, et al. Rates and risk factors for nonadherence to the medical regimen after adult solid organ transplantation. Transplantation 2007;83:858-73.

12. Pashaee N, Bouamar R, Hesselink DA, et al. CYP3A5 genotype is not related to the intrapatient variability of tacrolimus clearance. Ther Drug Monit 2011;33:369-71.

13. Staatz CE, Goodman LK, Tett SE, et al. Effect of CYP3A and ABCB1 single nucleotide polymorphisms on the pharmacokinetics and pharmacodynamics of calcineurin inhibitors: Part I. Clin Pharmacokinet 2010;49:141-75.

14. Elens $L$, Bouamar $R$, Hesselink DA, et al. A new functional CYP3A4 intron 6 polymorphism significantly affects tacrolimus pharmacokinetics in kidney transplant recipients. Clin Chem 2011;57:1574-83.

15. Waiser J, Slowinski T, Brinker-Paschke A, et al. Impact of the variability of cyclosporin $A$ trough levels on long-term renal allograft function. Nephrol Dial Transplant 2002;17:1310-7.

16. Hsiau M, Fernandez HE, Gjertson D, et al. Monitoring nonadherence and acute rejection with variation in blood immunosuppressant levels in pediatric renal transplantation. Transplantation 2011;92:918-22.

17. Whalen HR, Glen JA, Harkins V, et al. High intrapatient tacrolimus variability is associated with worse outcomes in renal transplantation using a low-dose tacrolimus immunosuppressive regime. Transplantation 2017;101:430-6.

18. Shuker N, van Gelder T, Hesselink DA, et al. Intra-patient variability in tacrolimus exposure: causes, consequences for clinical management. Transplant Rev 2015;29:78-84.

19. Sapir-Pichhadze R, Wang $Y$, Famure O, et al. Time-dependent variability in tacrolimus trough blood levels is a risk factor for late kidney transplant failure. Kidney Int 2014;85:1404-11.

20. Borra LC, Roodnat JI, Kal JA, et al. High within-patient variability in the clearance of tacrolimus is a risk factor for poor long-term outcome after kidney transplantation. Nephrol Dial Transplant 2010;25:2757-63. 